\title{
The SU(3) bosons and the spin nematic state on the spin-1 bilinear-biquadratic triangular lattice
}

\author{
Peng Li ${ }^{1}$, Guang-Ming Zhang ${ }^{2}$, and Shun-Qing Shen ${ }^{1}$, \\ ${ }^{1}$ Department of Physics, and Center for Theoretical and Computational Physics, \\ The University of Hong Kong, Pokfulam Road, Hong Kong, China and \\ ${ }^{2}$ Department of Physics and Center for Advanced Study, Tsinghua University, Beijing 100084, China.
}

(Dated: May 3, 2021)

\begin{abstract}
A bond-operator mean-field theory in the $\mathrm{SU}(3)$ bosons representation is developed to describe the antiferro-nematic phase of the spin-1 bilinear-biquadratic model. The calculated static structure factors reveal delicately that the antiferro-nematic state may exhibit both the ferro- and antiferroquadruple long-range orders, which is reminiscent of the ferrimagnets or the canted antiferromagnets. This result may influence the spin wave theory concerned with this phase. Possible relevance of this unconventional state to the quasi-two-dimensional triangular material $\mathrm{NiGa}_{2} \mathrm{~S}_{4}$ is addressed.

PACS numbers: 03.75.Mn, 75.10.Jm, 75.10.-b.
\end{abstract}

\section{INTRODUCTION}

The spin-1 bilinear-biquadratic model (SBBM)

$$
H=J_{\varphi} \sum_{\langle i j\rangle}\left[\cos \varphi \mathbf{S}_{i} \cdot \mathbf{S}_{j}+\sin \varphi\left(\mathbf{S}_{i} \cdot \mathbf{S}_{j}\right)^{2}\right]
$$

was put forward long time agc ${ }^{1.2 .3}$, where $\mathbf{S}_{i}$ is the spin1 operator. In one dimension, the phase diagram was established ${ }^{4,5,6.7}$, but there are still some controversies 7.8 . The phase diagram in two dimensions and above may be simpler because of suppression of quantum fluctuations. Generally speaking, there are two regimes exhibiting different types of spin nematic orders: (1) the ferro-nematic phase for $-3 \pi / 4<\varphi<-\pi / 2$ (2) the antiferro-nematic phase for $\pi / 4<\varphi<\pi / 2$. Recently the first regime with ferro-quadruple long-range order (LRO) attracts much attention due to the fact that the Mott insulating state was realized in a system of bosonic atoms in an optical lattice 9.10 .11 . Here we shall study the second regime by a bond-operator mean-field theory in $\mathrm{SU}(3)$ bosons representation. The unconventional properties of this nematic state, such as the absence of magnetic LRO and the gapless excitation, are quite instructive for explaining recent experimental observations in $\mathrm{NiGa}_{2} \mathrm{~S}_{4}{ }_{12,13,14}$.

In a framework of frustrated $\mathrm{SU}(N)$ model, we expressed the SBBM in terms of $\mathrm{SU}(3)$ generators and proposed an associated bond-operator mean-field theory in both bosonic and fermionic representations ${ }^{15,16}$. The theory is a generalization of the widely used Schwingerboson mean-field theory (SBMFT) ${ }^{17}$. The advantage of the theory is that we can use it to study either the ordered or disordered phases. In this paper, we shall use the bosonic theory to study the unconventional orders of the antiferro-nematic states on the triangular lattice. It will be shown that the ferro- and antiferro-quadruple LRO's may coexist at low temperature for the quadruple operators, which is reminiscent of the ferrimagnets or the canted antiferromagnets. And the uniform quadruple moments may keep nonzero at finite temperatures. These two new features enrich our knowledge of the antiferro- nematic state of this model. To show the relevance of this state to the observations in $\mathrm{NiGa}_{2} \mathrm{~S}_{4}$, we also calculate the physical quantities, e.g. the ground energy, the specific heat, and the uniform magnetic susceptibility. A similar theory with a different scheme had been applied to the ferro-nematic phase by one of the authors in a previous work ${ }^{8}$.

The paper is organized as follows. In Sec. II we introduce the $\mathrm{SU}(3)$ boson representation for spin 1 system, and express the Hamiltonian of Eq. (1) in terms of SU(3) generators. In Sec. III, we present the formalism of the bond-operator mean-field theory in bosonic language. Then in Sec. IV, we work out the mean-field equations and uncover some properties of the antiferronematic phase. In Sec. V, we present discussions of our results.

\section{SU(3) BOSONS REPRESENTATION}

In SBBM, each site has three states, $\left|m_{\alpha}\right\rangle$ with $m_{1}=$ $-1, m_{2}=0$, and $m_{3}=+1$, according to the eigenvalues of the $z$-component of spin, $S^{z}$. We reorganize the three states and introduce three bosonic creation operators,

$$
\begin{aligned}
b_{1}^{\dagger}|0\rangle & =\frac{1}{\sqrt{2}}\left(\left|m_{1}\right\rangle-\left|m_{3}\right\rangle\right), \\
b_{2}^{\dagger}|0\rangle & =\frac{i}{\sqrt{2}}\left(\left|m_{1}\right\rangle+\left|m_{3}\right\rangle\right), \\
b_{3}^{\dagger}|0\rangle & =\left|m_{2}\right\rangle .
\end{aligned}
$$

In terms of $b$ operators, the eight generators of $\mathrm{SU}(3)$ group can be expressed by three boson operators. They can be divided into two categories, the spin operators

$$
\begin{aligned}
& S_{i}^{x}=-i\left(b_{i 2}^{\dagger} b_{i 3}-b_{i 3}^{\dagger} b_{i 2}\right), \\
& S_{i}^{y}=-i\left(b_{i 3}^{\dagger} b_{i 1}-b_{i 1}^{\dagger} b_{i 3}\right), \\
& S_{i}^{z}=-i\left(b_{i 1}^{\dagger} b_{i 2}-b_{i 2}^{\dagger} b_{i 1}\right),
\end{aligned}
$$


and the quadrupole operators (second-order spin moments)

$$
\begin{aligned}
& Q_{i}^{(0)}=\left(S_{i}^{z}\right)^{2}-\frac{2}{3}=\frac{1}{3}\left(b_{i 1}^{\dagger} b_{i 1}+b_{i 2}^{\dagger} b_{i 2}-2 b_{i 3}^{\dagger} b_{i 3}\right), \\
& Q_{i}^{(2)}=\left(S_{i}^{x}\right)^{2}-\left(S_{i}^{y}\right)^{2}=-\left(b_{i 1}^{\dagger} b_{i 1}-b_{i 2}^{\dagger} b_{i 2}\right), \\
& Q_{i}^{x y}=S_{i}^{x} S_{i}^{y}+S_{i}^{y} S_{i}^{x}=-\left(b_{i 1}^{\dagger} b_{i 2}+b_{i 2}^{\dagger} b_{i 1}\right), \\
& Q_{i}^{y z}=S_{i}^{y} S_{i}^{z}+S_{i}^{z} S_{i}^{y}=-\left(b_{i 2}^{\dagger} b_{i 3}+b_{i 3}^{\dagger} b_{i 2}\right), \\
& Q_{i}^{z x}=S_{i}^{z} S_{i}^{x}+S_{i}^{x} S_{i}^{z}=-\left(b_{i 3}^{\dagger} b_{i 1}+b_{i 1}^{\dagger} b_{i 3}\right) .
\end{aligned}
$$

In this case the Hamiltonian, Eq. (1), can be expressed in terms of these generators and has a form of the generalized frustrated $\mathrm{SU}(3)$ model $^{16}$,

$H=\sum_{\langle i j\rangle} \Upsilon_{1}(i, j)+\sum_{\langle i j\rangle} \Upsilon_{2}(i, j)+\sum_{i} \lambda_{i}\left(\sum_{\mu} b_{i \mu}^{\dagger} b_{i \mu}-1\right)$,

where

$$
\begin{gathered}
\Upsilon_{1}(i, j)=J_{1} \sum_{\mu \nu} \mathcal{J}_{\nu}^{\mu}\left(r_{i}\right) \mathcal{J}_{\mu}^{\nu}\left(r_{j}\right), \\
\Upsilon_{2}(i, j)=-J_{2} \sum_{\mu \nu} \mathcal{J}_{\nu}^{\mu}\left(r_{i}\right) \mathcal{J}_{\nu}^{\mu}\left(r_{j}\right),
\end{gathered}
$$

with $J_{1}=J_{\varphi} \cos \varphi$ and $J_{2}=J_{\varphi}(\cos \varphi-\sin \varphi) . \mathcal{J}_{\nu}^{\mu}\left(r_{i}\right)=$ $b_{i \mu}^{\dagger} b_{i \nu}$ are the generators of the $\mathrm{SU}(3)$ group, and the Lagrangian multipliers $\lambda_{i}$ are introduced to realize the single occupancy of the bosons at each lattice site. The first term in Eq. (5) possesses the $\mathrm{SU}(3)$ symmetry because the operator,

$$
\sum_{\mu \nu} \mathcal{J}_{\nu}^{\mu}\left(r_{i}\right) \mathcal{J}_{\mu}^{\nu}\left(r_{j}\right) \equiv P_{i j}
$$

serves as the permutation operator, which swaps two quantum states at sites $i$ and $j$,

$$
P_{i j}|i, \mu ; j, \nu\rangle=|i, \nu ; j, \mu\rangle .
$$

The second term in Eq.(5) breaks the $\mathrm{SU}(N)$ symmetry on the triangular lattice.

\section{MEAN-FIELD THEORY}

\section{A. Decomposition Scheme}

Now we concentrate on the regime with $J_{1}>0$ and $J_{2}<0$. In the boson representation, we introduce two types of bond operators

$$
\begin{aligned}
& \Delta_{i j, \mu \nu}=b_{j \mu} b_{i \nu}-b_{j \nu} b_{i \mu}, \quad(\mu<\nu), \\
& \Xi_{i j, \mu \nu}=b_{j \mu}^{\dagger} b_{i \nu}-b_{j \nu}^{\dagger} b_{i \mu},(\mu<\nu),
\end{aligned}
$$
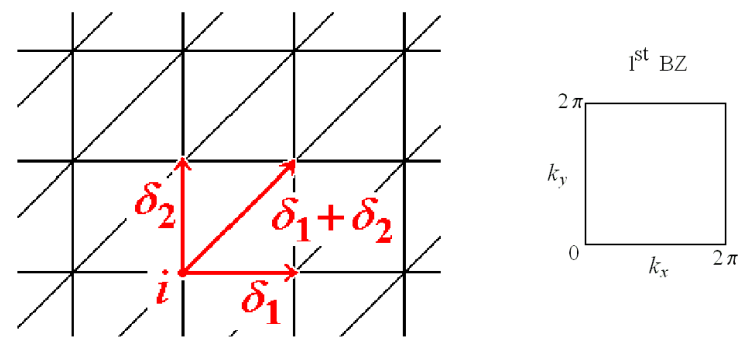

FIG. 1: (Color online) The lattice we used in the calculation, which is topologically equivalent to the triangular lattice given that the interactions along directions, $\delta_{1}, \delta_{2}$, and $\delta_{1}+\delta_{2}$, are equal. On this lattice, the first Brillouin zone is a square with volume $(2 \pi)^{2}$.

and the four-operator terms in the Hamiltonian Eq. (5) can be written as

$$
\begin{aligned}
& \Upsilon_{1}(i, j)=-J_{1} \sum_{\mu<\nu} \Delta_{i j, \mu \nu}^{\dagger} \Delta_{i j, \mu \nu}+J_{1}, \\
& \Upsilon_{2}(i, j)=-\left|J_{2}\right| \sum_{\mu<\nu}: \Xi_{i j, \mu \nu}^{\dagger} \Xi_{i j, \mu \nu}:+\left|J_{2}\right| .
\end{aligned}
$$

Notice the single occupancy constraint, $\sum_{\mu} b_{i \mu}^{\dagger} b_{i \mu}=1$, is used when the expressions are deduced. Consider that the model is isotropic, one can introduce two real meanfield parameters

$$
\begin{aligned}
& \Delta=\left\langle\Delta_{i j, \mu \nu}\right\rangle=\left\langle\Delta_{i j, \mu \nu}^{\dagger}\right\rangle, \\
& \Xi=\left\langle\Xi_{i j, \mu \nu}\right\rangle=\left\langle\Xi_{i j, \mu \nu}^{\dagger}\right\rangle .
\end{aligned}
$$

The prescribed mean fields represent the ultrashort-range correlations. However, when the mean field equations are solved, some physical restrictions should be fulfilled. For instance, the biquadratic term in the $\operatorname{SBBM}(N=3)$ can be written as

$$
\left(\mathbf{S}_{i} \cdot \mathbf{S}_{j}\right)^{2}=-\sum_{\mu<\nu}: \Xi_{i j, \mu \nu}^{\dagger} \Xi_{i j, \mu \nu}:+1,(\mu, \nu=1,2,3) .
$$

Since $\left(\mathbf{S}_{i} \cdot \mathbf{S}_{j}\right)^{2} \geqslant 0$, one would obtain the restriction

$$
\Xi=\left\langle\Xi_{i j, \mu \nu}\right\rangle \leqslant \frac{1}{\sqrt{3}} .
$$

Our numerical result shows that this restriction is well satisfied.

\section{B. Mean-Field Equations}

We limit our calculation on the triangular lattice (see Fig. (1).

The Hubbard-Stratonovich transformation is performed to decouple the Hamiltonian Eq. (5) into a bilin- 
ear form

$$
\begin{aligned}
H & =-J_{1} \Delta \sum_{\mu<\nu} \sum_{i, \delta>0}\left(\Delta_{i, i+\delta ; \mu \nu}+\Delta_{i, i+\delta ; \mu \nu}^{\dagger}\right) \\
& -\left|J_{2}\right| \Xi \sum_{\mu<\nu} \sum_{i, \delta}\left(\Xi_{i, i+\delta ; \mu \nu}+\Xi_{i, i+\delta ; \mu \nu}^{\dagger}\right) \\
& +\frac{z}{2} N_{\Lambda} N\left(J_{1} \Delta^{2}+\left|J_{2}\right| \Xi^{2}\right)+\lambda \sum_{i} \sum_{\mu} b_{i \mu}^{\dagger} b_{i \mu}-\lambda N_{\Lambda},
\end{aligned}
$$

where $\sum_{\delta>0}$ means summation over the nearest neighbours in the positive directions of a given site, $N_{\Lambda}$ is the total number of lattice sites, $z$ is the coordinate number of the lattice, e.g. $z=6$ for the triangular lattice.

After performing the Fourier transform and introducing the Nambu spinor in the momentum space,

$$
\Phi_{k}^{\dagger}=\left(b_{k, 1}^{\dagger}, b_{k, 2}^{\dagger}, b_{k, 3}^{\dagger}, b_{-k, 1}, b_{-k, 2}, b_{-k, 3}\right) .
$$

one can arrive at the mean-field Hamiltonian for the spin $S$ chain in a compact form,

$$
H=\frac{1}{2} \sum_{k} \Phi_{k}^{\dagger} M_{k} \Phi_{k}+\varepsilon_{0},
$$

where

$$
\begin{aligned}
M_{k} & =\lambda \sigma^{0} \otimes A_{0}+i \Delta_{k} \sigma^{x} \otimes A_{1}+i \Xi_{k} \sigma^{0} \otimes A_{1}, \\
A_{0} & =\left(\begin{array}{lll}
1 & 0 & 0 \\
0 & 1 & 0 \\
0 & 0 & 1
\end{array}\right), A_{1}=\left(\begin{array}{ccc}
0 & -1 & -1 \\
1 & 0 & -1 \\
1 & 1 & 0
\end{array}\right), \\
\Delta_{k} & =2 J_{1} \Delta \eta_{k}, \Xi_{k}=2\left|J_{2}\right| \Xi \eta_{k}, \eta_{k}=\sum_{\delta>0} \sin k_{\delta} \\
\varepsilon_{0} & =\frac{3}{2} z N_{\Lambda}\left(J_{1} \Delta^{2}+\left|J_{2}\right| \Xi^{2}\right)-\frac{5}{2} \lambda N_{\Lambda} .
\end{aligned}
$$

By diagonalizing the Hamiltonian, we get three spectra,

$$
\begin{aligned}
& \omega_{1}(k)=\lambda, \\
& \omega_{2}(k)=\sqrt{\left(\lambda-\sqrt{3} \Xi_{k}\right)^{2}-\left(\sqrt{3} \Delta_{k}\right)^{2}}, \\
& \omega_{3}(k)=\sqrt{\left(\lambda+\sqrt{3} \Xi_{k}\right)^{2}-\left(\sqrt{3} \Delta_{k}\right)^{2}} .
\end{aligned}
$$

Notice that the two spectra $\omega_{3}(k)$ and $\omega_{2}(k)$ has a relation of $\omega_{3}(-k)=\omega_{2}(k)$. By optimization of the total free energy

$$
F=\varepsilon_{0}-\frac{1}{\beta} \sum_{k, \mu} \ln \left[n_{B}\left(\omega_{\mu}\right)\left(n_{B}\left(\omega_{\mu}\right)+1\right)\right],
$$

where $n_{B}\left(\omega_{\mu}\right)$ is the Boltzmann distribution function, three mean-field equations are established

$$
\begin{gathered}
2-n_{B}(\lambda)=\int \frac{d^{2} k}{(2 \pi)^{2}} \frac{1-\widetilde{\Xi} \eta_{k}}{\widetilde{\omega}_{2}(k)} \operatorname{coth} \frac{\beta \widetilde{\omega}_{2}(k)}{2}, \\
\Delta=\frac{1}{3 \sqrt{3}} \int \frac{d^{2} k}{(2 \pi)^{2}} \frac{\widetilde{\Delta} \eta_{k}^{2}}{\widetilde{\omega}_{2}(k)} \operatorname{coth} \frac{\beta \widetilde{\omega}_{2}(k)}{2} \\
\Xi=\frac{1}{3 \sqrt{3}} \int \frac{d^{2} k}{(2 \pi)^{2}} \frac{\left(1-\widetilde{\Xi} \eta_{k}\right) \eta_{k}}{\widetilde{\omega}_{2}(k)} \operatorname{coth} \frac{\beta \widetilde{\omega}_{2}(k)}{2},
\end{gathered}
$$

in which we have introduced dimensionless quantities for convenience of calculation

$$
\begin{aligned}
\widetilde{\omega}_{\mu}(k) & =\frac{\omega_{\mu}(k)}{\lambda}, \\
\widetilde{\Delta} & =\frac{2 \sqrt{3} J_{1} \Delta}{\lambda}, \\
\widetilde{\Xi} & =\frac{2 \sqrt{3}\left|J_{2}\right| \Xi}{\lambda} .
\end{aligned}
$$

And $\beta=1 / k_{B} T, k_{B}$ is the Boltzmann constant. There are generally three branches of valid solutions: (i) nonzero solution, $\Delta \neq 0$ and $\Xi \neq 0$; (ii) zero solution, $\Delta=0$ and $\Xi \neq 0$; (iii) zero solution, $\Delta \neq 0$ and $\Xi=0$. The one with the lowest energy is picked out as the physically realized state. At zero temperature, the per site ground energy has a simple form,

$$
\frac{E_{0}}{N_{\Lambda}}=-\frac{3}{2} z\left(J_{1} \Delta^{2}+\left|J_{2}\right| \Xi^{2}\right) .
$$

\section{Green's Function and Susceptibility}

In order to calculate the susceptibility we introduce the Matsubara Green's function in the form of a $6 \times 6$ matrix,

$G(k, \tau)=-\left\langle T_{\tau} \Phi_{k}(\tau) \Phi_{k}^{\dagger}(0)\right\rangle=\frac{1}{\beta} \sum_{n} G\left(k, i \omega_{n}\right) e^{-i \omega_{n} \tau}$.

The bosonic Matsubara Green's function $G\left(k, i \omega_{n}\right)$ is generally worked out as,

$$
G\left(k, i \omega_{n}\right)=\left(i \omega_{n} \sigma_{z} \otimes A_{0}-M_{k}\right)^{-1},
$$

where $\omega_{n}=2 n \pi / \beta$. The three spectra, Eq. (19), can also be read out from the poles of the Green's function.

As we shall study spin order as well as the nematic order in the system, we define two types of correlation functions in Matsubara formalism. The first type is the spin-spin correlation. Due to rotational invariance, we need only to consider the imaginary-time spin-spin correlation for $S^{z}$,

$$
\chi_{S^{z}}(q, \tau)=\left\langle T_{\tau} S^{z}(q, \tau) S^{z}(-q, 0)\right\rangle .
$$

Its Fourier transform is given by

$$
\chi_{S^{z}}\left(q, i \omega_{n}\right)=\int_{0}^{\beta} d \tau e^{i \omega_{n} \tau} \chi_{S^{z}}(q, \tau) .
$$


The second type is the imaginary-time quadrupolequadrupole correlation and its Fourier transform defined for the quadrupole operators $Q$ 's in Eq. (4) is given by,

$$
\begin{aligned}
\chi_{Q}(q, \tau) & =\left\langle T_{\tau} Q(q, \tau) Q(-q, 0)\right\rangle, \\
\chi_{Q}\left(q, i \omega_{n}\right) & =\int_{0}^{\beta} d \tau e^{i \omega_{n} \tau} \chi_{Q}(q, \tau), \\
Q & \in\left\{Q^{(0)}, Q^{(2)}, Q^{x y}, Q^{y z}, Q^{z x}\right\} .
\end{aligned}
$$

Due to the rotational invariance, here we only present two of them, $\chi_{Q^{(2)}}$ and $\chi_{Q^{x y}}\left(\chi_{Q^{(0)}}\right.$ is equivalent to $\chi_{Q^{(2)}}$, $\chi_{Q^{y z}}$ and $\chi_{Q^{z x}}$ are equivalent to $\left.\chi_{Q^{x y}}\right)$. According to the single-mode approximation theory ${ }^{18}, \chi_{S^{z}}$ is related to the spin order by the single mode $S^{z}(q)|0\rangle$ with spin density wave

$$
S^{z}(q)=\sum_{i} e^{i q \cdot R_{i}} S_{i}^{\alpha}
$$

while $\chi_{Q}$ is related to the nematic order by the single mode $Q(q)|0\rangle$ with quadrupole density wave

$$
Q(q)=\sum_{i} e^{i q \cdot R_{i}} Q_{i}
$$

The expressions of the susceptibilities at zero temperature can be found in Appendix. A.

\section{LONG-RANGE SPIN NEMATIC ORDER ON TRIANGULAR LATTICE}

The non-zero solution of the mean field parameters satisfies

$$
\widetilde{\Delta}+\widetilde{\Xi}=\frac{2}{3 \sqrt{3}},
$$

at zero temperature and on the triangular lattice. With this relation, the spectrum $\omega_{2}(k)$ becomes gapless at the point,

$$
k^{*}=\left(k_{x}^{*}, k_{y}^{*}\right)=\left(\frac{\pi}{3}, \frac{\pi}{3}\right)
$$

where the boson condensation occurs. As temperature becomes nonzero, the spectrum $\omega_{2}(k)$ will open a gap and thus no condensation occurs. When the condensation occurs, we should parse the condensation terms and rewrite the equations as

$$
\begin{aligned}
\rho_{0} & =2-\int \frac{d^{2} k}{(2 \pi)^{2}} \frac{1-\widetilde{\Xi} \eta_{k}}{\widetilde{\omega}_{2}(k)}, \\
\Delta & =\frac{1}{2} \rho_{0}+\frac{1}{3 \sqrt{3}} \int \frac{d^{2} k}{(2 \pi)^{2}} \frac{\widetilde{\Delta} \eta_{k}^{2}}{\widetilde{\omega}_{2}(k)} \\
\Xi & =\frac{1}{2} \rho_{0}+\frac{1}{3 \sqrt{3}} \int \frac{d^{2} k}{(2 \pi)^{2}} \frac{\left(1-\widetilde{\Xi} \eta_{k}\right) \eta_{k}}{\widetilde{\omega}_{2}(k)},
\end{aligned}
$$

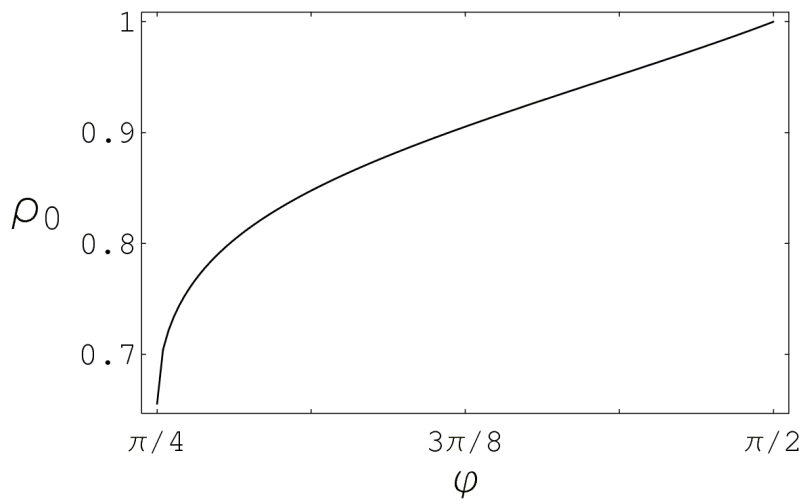

FIG. 2: The condensation density $\rho_{0}$ at zero temperature.

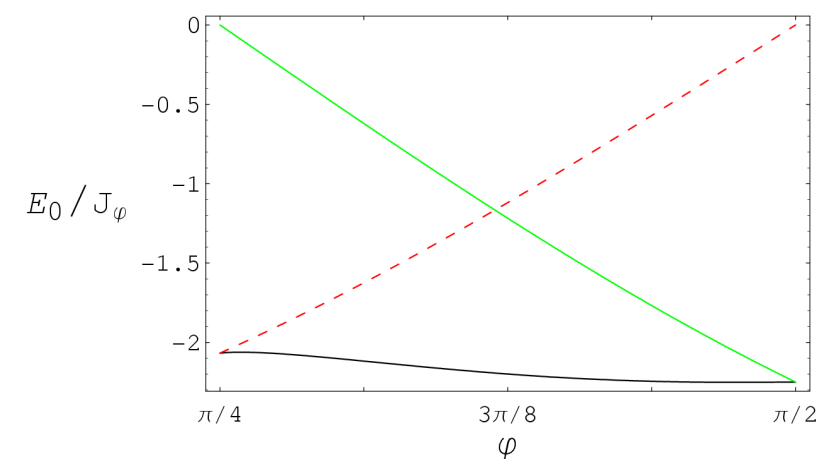

FIG. 3: (Color online) The ground energies. The solid line is the non-zero solution: $\Delta \neq 0, \Xi \neq 0$. The dashed line is the zero solution: $\Delta \neq 0, \Xi=0$. The dotted line is the zero solution: $\Delta=0, \Xi \neq 0$. This figure shows the non-zero solution is the optimized one.

where the condensation density is (see numerical result in Fig. 2)

$$
\rho_{0}=\left[\frac{2 n_{B}\left(\widetilde{\omega}_{2}\left(k^{*}\right)\right)+1}{N_{\Lambda} \widetilde{\omega}_{2}\left(k^{*}\right)}\right] \frac{3 \sqrt{3} \widetilde{\Delta}}{2} .
$$

From the ground energies shown in Fig. [3

we see the non-zero solution is the optimized one in the range $\pi / 4<\varphi<\pi / 2$. At the $\mathrm{SU}(3)$ point of $\varphi=\pi / 4$, the zero solution with $\Delta \neq 0$ and $\Xi=0$ is degenerate with the non-zero solution. At the point of $\varphi=\pi / 2$, the zero solution with $\Delta=0$ and $\Xi \neq 0$ is degenerate with the non-zero solution. This reflects the fact that the two points are highly symmetric points. In the range $\pi / 4<\varphi<\pi / 2$, the condensate is non-zero. So what is the physical effect of the quasiparticle condensation? By probing the possible orders in the system, we find the condensation leads to the nematic LRO while spin moments vanish, i.e. the nematic state is non-magnetic. This conclusion is drawn from the static spin and quadrupole structure factors shown in Fig. 4 (Please refer to the expressions listed in Appendix. A.)

The static quadrupole structure factor $\chi_{Q^{(2)}}\left(q, \tau=0^{+}\right)$ 

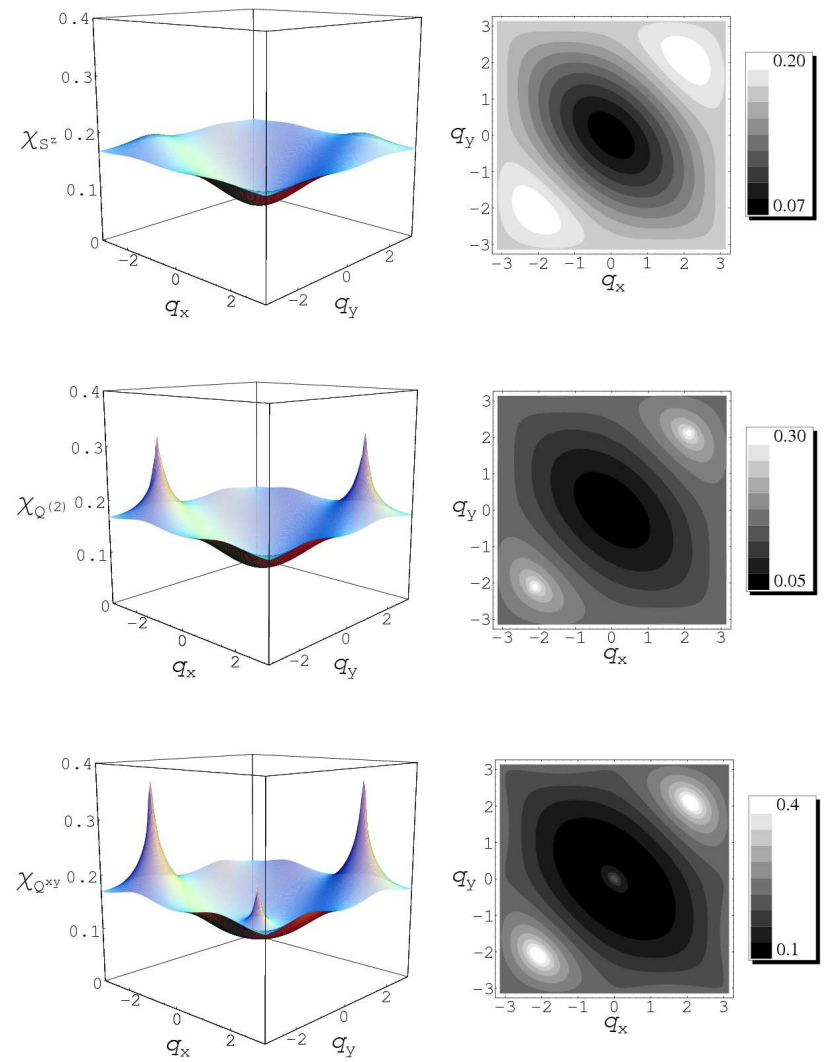

FIG. 4: (Color online) Static structure factors $\chi_{S^{z}}, \chi_{Q^{(2)}}$, and $\chi_{Q^{x y}}$ at a sampled point $\varphi=0.863938$. The images at other point in the regime $\frac{\pi}{4}<\varphi<\frac{\pi}{2}$ are qualitatively the same.

and $\chi_{Q^{x y}}\left(q, \tau=0^{+}\right)$show sharp divergent peaks at the two points $q^{*}= \pm 2 k^{*}= \pm\left(\frac{2 \pi}{3}, \frac{2 \pi}{3}\right)$ indicating the existence of antiferro-quadrupole LRO. While the static spin structure factor $\chi_{S^{z}}\left(q, \tau=0^{+}\right)$shows two small humps at $q^{*}= \pm\left(\frac{2 \pi}{3}, \frac{2 \pi}{3}\right)$. Surprisingly $\chi_{Q^{x y}}\left(q, \tau=0^{+}\right)$also exhibits a divergent peak at the point $q^{0}=(0,0)$. Analytically, at the condensate points, the divergent terms of $\chi_{Q^{x y}}$ are parsed out as (obtained by taking the limit $T \rightarrow 0$ in the finite temperature results)

$$
\begin{aligned}
\chi_{Q^{x y}}^{\rho_{0}}\left(q^{0}\right) & =\frac{1}{9} \rho_{0}^{2} N_{\Lambda}, \\
\chi_{Q^{x y}}^{\rho_{0}}\left(q^{*}\right) & =\frac{2}{9} \rho_{0}^{2} N_{\Lambda} .
\end{aligned}
$$

The ratio of the weights of the ferro- and antiferroquadrupole divergent peaks is

$$
r=\frac{\chi_{Q^{x y}}^{\rho_{0}}\left(q^{0}\right)}{\chi_{Q^{x y}}^{\rho_{0}}\left(q^{*}\right)}=\frac{1}{2} .
$$

$\chi_{Q^{x y}}^{\rho_{0}}\left(q^{0}\right)$ and $\chi_{Q^{x y}}^{\rho_{0}}\left(q^{*}\right)$ are proportional to the number of the lattice site $N_{\Lambda}$, which indicates that the ferro- and antiferro-quadrupole LRO coexist for $Q_{i}^{x y}$. The result can be understood well if one notices that the states de- fined in Eq. (2) are eigenstates of $Q_{i}^{(2)}$,

$$
\begin{gathered}
\left|Q_{i}^{(2)}=-1\right\rangle=b_{i 1}^{\dagger}|0\rangle, \\
\left|Q_{i}^{(2)}=1\right\rangle=b_{i 2}^{\dagger}|0\rangle, \\
\left|Q_{i}^{(2)}=0\right\rangle=b_{i 3}^{\dagger}|0\rangle .
\end{gathered}
$$

If on a bipartite lattice, $\left|Q_{i}^{(2)}=-1\right\rangle$ and $\left|Q_{i}^{(2)}=1\right\rangle$ would align in a staggered pattern with antiferroquadrupole LRO in the classical point of view. While on the triangular lattice, the LRO arrangement of quadrupole moments, $\left\langle Q_{i}^{(2)}\right\rangle$, is a $2 \pi / 3$ structure as we revealed above. Nevertheless they are not the eigenstates of $Q_{i}^{x y}$, instead

$$
\begin{gathered}
\left|Q_{i}^{(2)}=-1\right\rangle=\frac{1}{\sqrt{2}}\left(\left|Q_{i}^{x y}=1\right\rangle-\left|Q_{i}^{x y}=-1\right\rangle\right), \\
\left|Q_{i}^{(2)}=1\right\rangle=\frac{-1}{\sqrt{2}}\left(\left|Q_{i}^{x y}=1\right\rangle+\left|Q_{i}^{x y}=-1\right\rangle\right) .
\end{gathered}
$$

So the antiferro-quadrupole moment of $Q_{i}^{(2)}$ means the existence of both the ferro- and antiferro-quadrupole moments of $Q_{i}^{x y}$. This phenomenon is reminiscent of the ferrimagnets or the canted anteferromagnets, which exhibit both ferromagnetic and antiferromagnetic orders.

Now we address to the two terminals of the range $\pi / 4 \leqslant \varphi \leqslant \pi / 2$. At the $\mathrm{SU}(3)$ point $\varphi=\pi / 4$, the static spin structure factor also becomes divergent at $q^{*}= \pm\left(\frac{2 \pi}{3}, \frac{2 \pi}{3}\right)$. The state is degenerated with the nematic phase, which reflects the higher symmetry of the system $^{1.2}$. At $\varphi=\pi / 2$ where the Hamiltonian becomes $H=\sum_{i j}\left(\mathbf{S}_{i} \cdot \mathbf{S}_{j}\right)^{2}$, all of the static structure factors vanish and the system is free of both spin and quadrupole moments, which means the system is totally disordered.

The Matsubara formalism facilitates the evaluation of the expectation values at finite temperatures $(\pi / 4<\varphi<$ $\pi / 2)$

$$
\begin{aligned}
& \left\langle b_{i 1}^{\dagger} b_{i 1}\right\rangle=\left\langle b_{i 2}^{\dagger} b_{i 2}\right\rangle=\frac{1}{3}, \\
& \left\langle b_{i 1}^{\dagger} b_{i 2}\right\rangle=\left\langle b_{i 2}^{\dagger} b_{i 1}\right\rangle=\frac{1}{6}-\frac{1}{2} n_{B}(\lambda) .
\end{aligned}
$$

Thus we obtain

$$
\begin{aligned}
\left\langle S_{i}^{z}\right\rangle & =0 \\
\left\langle Q_{i}^{(2)}\right\rangle & =0 \\
\left\langle Q_{i}^{x y}\right\rangle & =-\left[\frac{1}{3}-n_{B}(\lambda)\right] .
\end{aligned}
$$

The uniform quadrupole moment $\left\langle Q_{i}^{x y}\right\rangle$ keeps nonzero at finite temperatures. This result does not mean the finite-temperature phase transition, just shows a robust uniform quadrupole moment.

It is well known, for the noninteracting Bose gas in three dimensions, the density of states (DOS) will vanish 


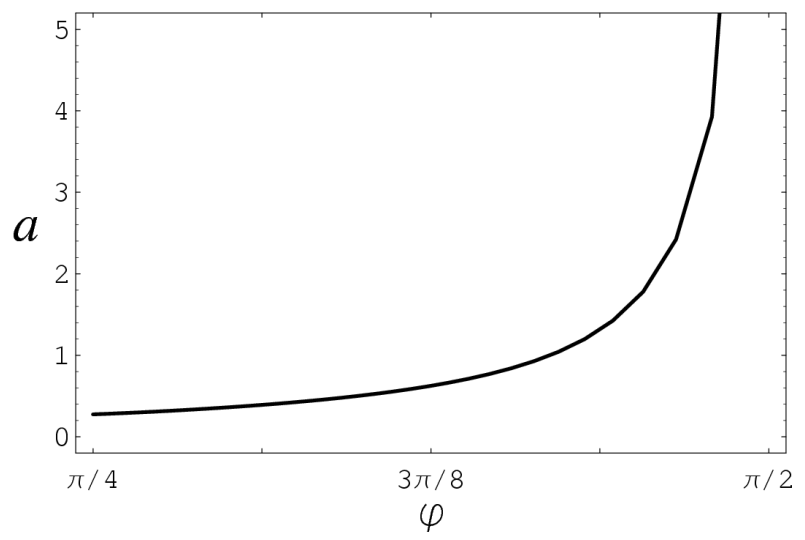

FIG. 5: The coefficient $a$ in Eq. (44).

with the energy decreasing down to zero, i.e. $D(E) \sim$ $\sqrt{E} \rightarrow 0$ when $E \rightarrow 0$, which leads to the Bose condensation at the gapless point $E=0$ at low temperatures. The situation is similar here. The DOS is defined by

$$
D(E)=\sum_{k, \mu} \delta\left(E-\omega_{\mu}(k)\right) .
$$

Since there exist a flat band $\omega_{1}=\lambda$ for quasi-particles, $D(E)$ always has a divergent peak at $E=\lambda$. We find that DOS rises linearly in $E$ from zero in the range of $\pi / 4 \leqslant \varphi<\pi / 2$,

$$
D(E) \sim a(\varphi) E+O\left(E^{2}\right),
$$

because the gapless spectrum $\omega_{2}(k)$ exhibits a node at $k^{*}=\left(\frac{\pi}{3}, \frac{\pi}{3}\right)$. By the DOS in Eq. (44), the low temperature specific heat is shown to exhibit the law of $T^{2}$,

$$
\begin{aligned}
C_{V} & =\int d E D(E) \frac{E}{2 k_{B} T}\left(\sinh \frac{E}{2 k_{B} T}\right)^{-2} \\
& \sim \frac{2 \pi^{2}}{3} a(\varphi) k_{B}^{2} T^{2} .
\end{aligned}
$$

The coefficient $a(\varphi)$ is plotted in Fig. 5

While at the terminal $\varphi=\pi / 2$, the node of the spectra disappears and the DOS has the form $D(E) \sim b+c E$ with $b \neq 0$, then one would get $C_{V} \sim \frac{\pi^{2}}{3} b k_{B} T$.

It is noteworthy that, at nonzero temperatures, the spectrum $\omega_{2}(k)$ is gapful and the DOS always has $D(E) \sim b+c E$. But at very low temperatures, $b$ is quite small and the power law in Eq. 455) can be satisfied asymptotically.

The uniform magnetic susceptibility at zero temperature is obtained by Kramers-Kronig relation ${ }^{18}$

$$
\chi_{M}=\lim _{q \rightarrow 0} \frac{1}{\pi} \int_{0}^{\infty} d \omega \frac{\operatorname{Im} \chi_{S^{z}}(q, \omega)}{\omega} .
$$

or by analytic continuation 19

$$
\chi_{M}=\lim _{q \rightarrow 0} \lim _{i \omega_{n} \rightarrow 0} \chi_{S^{z}}\left(q, i \omega_{n}\right),
$$

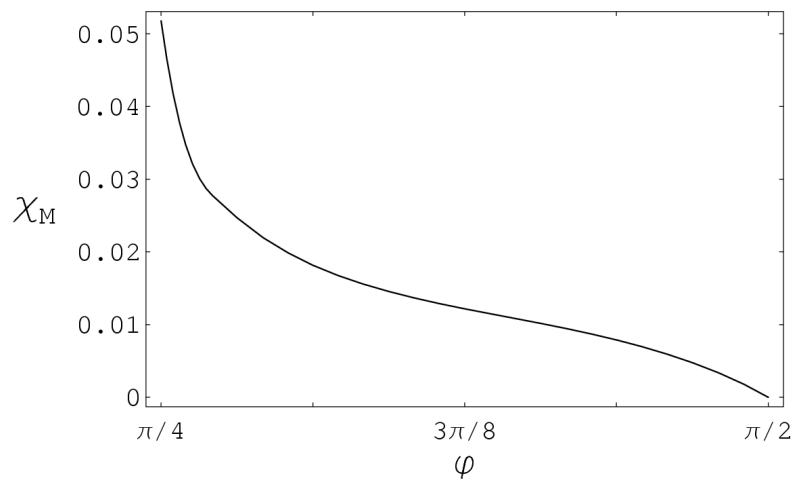

FIG. 6: The magnetic susceptibility $\chi_{M}$ at zero temperature.

They give the same result. At zero temperature, $\chi_{M}$ versus $\varphi$ is illustrated in Fig. 6]

$\chi_{M}$ reaches the maximal value at the $\mathrm{SU}(3)$ point $\varphi=$ $\pi / 4$, while it approaches zero at the end point $\varphi=\pi / 2$ where the system is inert to the external inspiration.

\section{DISCUSSION}

Recently the insulating antiferromagnet $\mathrm{NiGa}_{2} \mathrm{~S}_{4}$ arouses much attention ${ }^{12}$. The spin disorder observed in the experiment suggest that it may be a realization of the conceptual spin liquid that has long been explored over the past decades. This chalcogenide has a stacked triangular lattice with weak interactions between layers. Strong Hund's coupling in $\mathrm{Ni}^{2+}\left(t_{2 g}^{6} e_{g}^{2}\right)$ leads to the magnetism with spin $S=1$. Magnetic neutron scattering shows absence of conventional magnetic order and excludes the possibility of bulk spin glass freezing at low temperatures. Its specific heat shows low temperature power law, $C_{V} \sim T^{2}$, indicating gapless excitations and linearly dispersive modes in two dimensions. No divergence was observed for the magnetic susceptibility with the temperature decreasing down to $0.35 K$. These features can be produced by the nematic state as we studied above. However the incommensurate short-range order observed in the experiment still remains untouched.

Our bond-operator mean-field theory has the same origin as the SBMFT and is superior to the molecularfield approximation (MFA) because the MFA starts from a prescribed ferro- or antiferro-order and produces the same result regardless of the dimensionality ${ }^{2,20}$, while the our theory has no bias on the order or disorder of the ground state in advance. In two dimensions, we got a gapless nematic phase with quadruple LRO as we illustrated above for the triangular lattice. In one dimension, we got a gapped nematic phase ${ }^{21}$. Nevertheless, any mean-field theory can not be conclusive in its own right, thus other methods for the same problem are demanded. Unlike the spin wave theory, the bond-operator mean-field theory used in this paper does not prescribe 
an ordered state in advance. It has the same origin as SBMFT, except the species of bosons is altered from two to three. The antiferro-quadruple LRO emerges as a consequence of the condensation of the $\mathrm{SU}(3)$ bosons. Our results also show the quadruple operators are divided into two types. They have different LRO patterns and should be considered differently in a spin wave theory (i.e., for $Q^{(0)}$ and $Q^{(2)}$, one need only to consider their antiferroorders; while for $Q^{x y}, Q^{y z}$, and $Q^{z x}$, one should consider their ferro- and antiferro-orders at the same time. The coexistence of ferro- and antiferro-quadruple LRO's reveals that the quadruple operators can not be considered as the analogues of spin operators in the magnetic LRO phenomena. And as a merit of bosonic language, we expect that this theory gives good estimation of ground energy values (see Fig. 13), like the SBMFT 22.23 . To see how this theory describes the antiferromagnetic phase $(-\pi / 4<\varphi<\pi / 4)$ of the SBBM is also desirable, which will be considered in our future work.

In summary, a SU(3) bosons representation is introduced and the associated bond-operator mean-field theory is established to describe the antiferro-nematic phase of SBBM. It is revealed delicately that this nematic state may exhibit both the ferro- and antiferro-quadruple LRO's, which is reminiscent of the ferrimagnets or the canted antiferromagnets. And the quadruple LRO may survive to finite temperatures. The system may provide a rare example exhibiting finite-temperature phase transition in two dimensions. Possible relevance of this unconventional state to the quasi-two-dimensional triangular material $\mathrm{NiGa}_{2} \mathrm{~S}_{4}$ is addressed.

This work was supported by the Research Grant Council of Hong Kong under Grant No.: HKU7038/04P.

APPENDIX A: STATIC SPIN AND QUADRUPOLE STRUCTURE FACTORS

The static spin and quadrupole structure factors at zero temperature, $\chi\left(q, \tau=0^{+}\right)$, are worked out as

$$
\begin{aligned}
\chi_{S^{z}}\left(q, 0^{+}\right) & =\int \frac{d^{2} k}{(2 \pi)^{2}}\left[A_{2}(k)+A_{3}(k)\right. \\
& +3 A_{2}(k) B_{2}(k+q)+3 A_{3}(k) B_{3}(k+q) \\
& \left.+3 C_{2}(k) C_{2}(k+q)+3 C_{3}(k) C_{3}(k+q)\right],
\end{aligned}
$$

$$
\begin{aligned}
\chi_{Q^{(2)}}\left(q, 0^{+}\right) & =\int \frac{d^{2} k}{(2 \pi)^{2}}\left[A_{2}(k)+A_{3}(k)\right. \\
& +3 A_{2}(k) B_{3}(k+q)+3 A_{3}(k) B_{2}(k+q) \\
& \left.+3 C_{2}(k) C_{3}(k+q)+3 C_{3}(k) C_{2}(k+q)\right],
\end{aligned}
$$

$$
\begin{aligned}
\chi_{Q^{x y}}\left(q, 0^{+}\right) & =\int \frac{d^{2} k}{(2 \pi)^{2}}\left[\frac{1}{3} A_{2}(k)+\frac{1}{3} A_{3}(k)\right. \\
& +A_{2}(k) B_{2}(k+q)+A_{3}(k) B_{3}(k+q) \\
& +4 A_{2}(k) B_{3}(k+q)+4 A_{3}(k) B_{2}(k+q) \\
& +4 C_{2}(k) C_{3}(k+q)+4 C_{3}(k) C_{2}(k+q) \\
& \left.-C_{2}(k) C_{2}(k+q)-C_{3}(k) C_{3}(k+q)\right]
\end{aligned}
$$

where the abbreviated notations are

$$
\begin{aligned}
& A_{2}(k)=\frac{1}{6}\left[1+\frac{\lambda-\sqrt{3} \Xi_{k}}{\omega_{2}(k)}\right] \\
& A_{3}(k)=\frac{1}{6}\left[1+\frac{\lambda+\sqrt{3} \Xi_{k}}{\omega_{3}(k)}\right]=A_{2}(-k), \\
& B_{2}(k)=\frac{1}{6}\left[1-\frac{\lambda-\sqrt{3} \Xi_{k}}{\omega_{2}(k)}\right] \\
& B_{3}(k)=\frac{1}{6}\left[1-\frac{\lambda+\sqrt{3} \Xi_{k}}{\omega_{3}(k)}\right]=B_{2}(-k), \\
& C_{2}(k)=\frac{1}{6} \frac{\sqrt{3} \Delta_{k}}{\omega_{2}(k)}, \\
& C_{3}(k)=\frac{1}{6} \frac{\sqrt{3} \Delta_{k}}{\omega_{3}(k)}=-C_{2}(-k) .
\end{aligned}
$$

$\Xi_{k}$ and $\Delta_{k}$ can be found in Eq. (18c). Note that for $\chi_{Q^{(2)}}\left(q, 0^{+}\right)$and $\chi_{Q^{x y}}\left(q, 0^{+}\right)$, one should consider the divergent terms at the points $q^{*}= \pm\left(\frac{2 \pi}{3}, \frac{2 \pi}{3}\right)$ and $q^{0}=(0,0)$, such as Eq. (37). 
1 H. H. Chen and P. M. Levy, Phys. Rev. Lett. 27, 1383 (1971); ibid. 27, 1385 (1971).

${ }^{2}$ H. H. Chen and P. M. Levy, Phys. Rev. B 7, 4267 (1973).

3 N. Papanicolaou, Nucl. Phys. B 305, 367 (1988).

4 A. V. Chubukov, Phys. Rev. B 43, 3337 (1991).

${ }^{5}$ U. Schollwöck, Th. Jolicoeur, and T. Garel, Phys. Rev. B 53, 3304 (1996).

${ }^{6}$ G. Fáth and A. Suto, Phys. Rev. B 62, 3778 (2000).

7 A. Läuchli, G. Schmid, and S. Trebst, preprint, cond-mat/0607173

8 G. M. Zhang and L. Yu, preprint, cond-mat/0507158

9 M. Greiner et al., Nature 415, 39 (2002);

10 S.K. Yip, Phys. Rev. Lett. 90, 250402 (2003);

11 A. Imambekov, M. Lukin, and E. Demler, Phys. Rev. A 68, 063602 (2003); Phys. Rev. Lett. 93, 120405 (2004).

12 S. Nakatsuji, Y. Nambu, H. Tonomura, O. Sakai, S. Jonas, C. Broholm, H. Tsunetsugu, Y. Qiu, and Y. Maeno, Science 309, 1697 (2005).

${ }^{13} \mathrm{H}$. Tsunetsugu and M. Arikawa, preprint, cond-mat/0512209
14 A. Läuchli, F. Mila, and K. Penc, preprint, cond-mat/0605234

15 G. M. Zhang and S. Q. Shen, Phys. Rev. Lett. 87, 157201 (2001); S. Q. Shen and G. M. Zhang, Europhys. Lett. 57, 274 (2002).

16 P. Li and S. -Q. Shen, New J. Phys. 6, 160 (2004).

17 A. Auerbach and D. P. Arovas, Phys. Rev. Lett. 61, 617 (1988); D. P. Arovas and A. Auerbach Phys. Rev. B 38, 316 (1988).

18 A. Auerbach, Interacting Electrons and Quantum Magnetism, page 235 (Springer-Verlag, New York, 1994).

19 G. D. Mahan, Many-Particle Physics, second edition, page 227 (Kluwer Academic/Plenum, 2000).

20 K. Harada and N. Kawashima, Phys. Rev. B 65, 052403 (2002).

21 P. Li, G. -M. Zhang, and S. -Q. Shen, unpublished.

22 P. Li and S. -Q. Shen, Phys. Rev. B 72, 214439 (2005).

23 H. A. Ceccatto, C. J. Gazza, and A. E. Trumper, Phys. Rev. B 47, R12329 (1993). 